\title{
Imaging Stars by Performing Full-Stokes Optical Interferometric Polarimetry
}

\author{
Nicholas M. Elias II ${ }^{1 \dagger}$, Stanislav S. Edel ${ }^{2}$, Carol E. Jones ${ }^{3}$, Frances E. Mackay ${ }^{3}$, David \\ Mozurkewich $^{4}$, Anders M. Jorgensen ${ }^{5}$, and Henrique R. Schmitt ${ }^{6}$
}

${ }^{1}$ Array Operations Center, National Radio Astronomy Observatory, Socorro, NM 87801-0387, USA

${ }^{2}$ Department of Physics, New Mexico Institute of Mining and Technology, Socorro, NM 87801, USA

${ }^{3}$ Department of Physics and Astronomy, University of Western Ontario, London, ON N6A 3K7, Canada

${ }^{4}$ Seabrook Engineering, Seabrook, MD 20706-3018, USA

${ }^{5}$ Department of Electrical Engineering, New Mexico Institute of Mining and Technology, Socorro, NM 87801, USA

${ }^{6}$ Computational Physics Incorporated, Springfield, VA 22151-2110, USA

Optical interferometry and polarimetry have separately provided new insights into stellar astronomy, especially in the fields of fundamental parameters and atmospheric models. We present: scientific justifications for "full-Stokes" optical interferometric polarimetry (OIP); updated instrument requirements; preliminary beam combiner designs; polarimeter design; end-to-end OIP data reduction; and realistic reimaged full-Stokes models of Be stars with a suitable number of telescopes plus noise sources. All of this work represents preliminary research to construct an OIP beam combiner.

Keywords: optical system design, optical interferometry, optical polarimetry, imaging with optical interferometers and polarimeters

\section{INTRODUCTION}

Optical interferometry has benefitted significantly from increased sensitivity, spatial resolution, and spectral resolution. Similarly, the capabilities of optical polarimeters have also improved greatly in terms of sensitivity and spectral resolution. The next logical step is to combine long-baseline optical interferometry with polarimetry, which is called full-Stokes optical interferometric polarimetry (OIP; Elias et al. 2008).

Full-Stokes OIP beam combiners can be added to existing optical interferometers to obtain simple yet significant results (Elias 2004b). CHARA has an OIP beam combiner that is undergoing testing (Mourard et al. 2008), but it is not a full-Stokes instrument. Design and operational experience with a simple full-Stokes OIP instrument will benefit the next generation of interferometers for OIP as well as scalar ( Stokes I) observations with improved calibration.

In this paper, we present scientific projects to demonstrate the benefits of OIP. They represent a small subset of many others. We present updated requirements and a preliminary full-Stokes beam combiner designs. One employs simple optics and a sequential polarimeter, while the other is a bit more complicated and includes a simultaneous polarization capability. We also discuss a preliminary end-to-end design for data reduction, including coherent averaging, calibration, and imaging. In addition, we present a reimaged Be star model, employing a realistic number of telescopes and noise, based on part of the data-reduction design. (c) This is an Open Access article distributed under the terms of the Creative Commons Attribution Non-Commercial License (http://creativecommons.org/licenses/by-nc/3.0/) which permits unrestricted non-commercial use, distribution, and reproduction in any medium, provided the original work is properly cited.
Received Dec 02, 2011 Revised Jan 19, 2012 Accepted Feb 02, 2012 ${ }^{\dagger}$ Corresponding Author

E-mail:nelias@nrao.edu

Tel: +1-575-835-7178 Fax: +1-575-835-7027 


\section{SCIENTIFIC JUSTIFICATIONS}

There are a number of possible OIP scientific projects (Elias et al. 2008) that involve scattering and/or magnetic interactions. In this paper, we highlight four of them.

Consider an early-type star with a spherically symmetric Thomson scattering atmosphere (Chandrasekhar 1950). Such stars are intrinsically unpolarized when observed with classical polarimeters because of azimuthal symmetry, and the magnitude of the polarization increases toward the limb (Fig. 1a). OIP breaks the symmetry (Elias 2004b), thus making even previously uninteresting calibrator stars interesting. The polarized visibilities provide better information on the stellar structure than Stokes I visibilities. There is very little difference between the three models for Stokes I visibilities (hard to measure), even for large spatial frequencies, but for linear Stokes visibilities the differences are quite clear (Fig. 1a).

Late-type stars exhibit magnetically induced polarization from Zeeman splitting. Such observations indicate how these objects produce magnetic fields in their interiors. Fully convective stars can create large-scale fields without differential rotation, which is inconsistent with existing models. Donati et al. (2006) mapped the magnetic fields of these stars with imaging tomography from polarization time series (Fig. 1b). The rotational phase versus velocity diagram in the Stokes $\mathrm{V}$ parameter show the positive and negative Zeeman components, which can be converted to tomographic model images of magnetic fields. OIP observations of the stellar surfaces will directly confirm Zeeman splitting measurements.

Just as with $\mathrm{OB}$ stars, the polarization of Be stars is also produced by Thomson scattering, but it comes from an axisymmetric disk orbiting the star. Classical polarimeters detect polarization from these objects at the $\sim 1 \%$ level due to the flattened disk geometry (as long as it is not viewed face on). H $\alpha$ emission, acting as a tracer of the disk shape and angular size, has been observed with classical optical interferometers (Quirrenbach et al. 1997). OIP measurements, however, will provide even more information because continuum radiation dominates the disk environment compared to line emission.

Mackay et al. (2009) has shown how synthesized visibilities of Be stars depend on parameters from a simple geometric model and a more detailed radiative transfer model. At $2.2 \mu \mathrm{m}$, the polarized Stokes visibilities (normalized) range from $10^{-2}-10^{-3}$ for short baselines and $10^{-3}-10^{-4}$ for long ones. In Fig. 2, we present sample visibilities from a scaled very large array (VLA)-like array using the Common Astronomy Software Applications (CASA) viewer task ${ }^{1}$.

Models for Be stars can be extended to interacting binary stars. For example, $\beta$ Lyr has a late B-type star los-

${ }^{1}$ Common Astronomical Software Applications (http://casa.nrao.edu), an interferometry image-processing package.

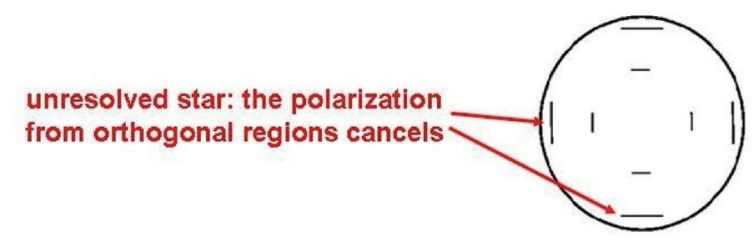

interferometry resolves the star, breaking the symmetry

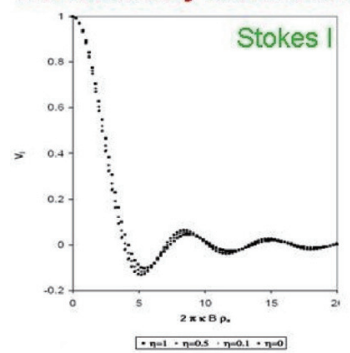

not so easy to distinguish

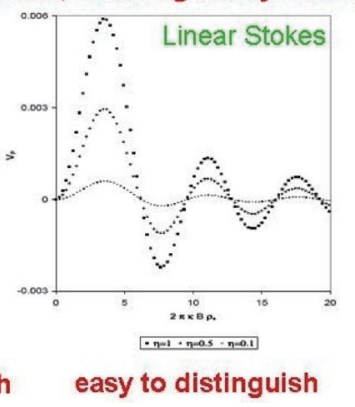

(a)

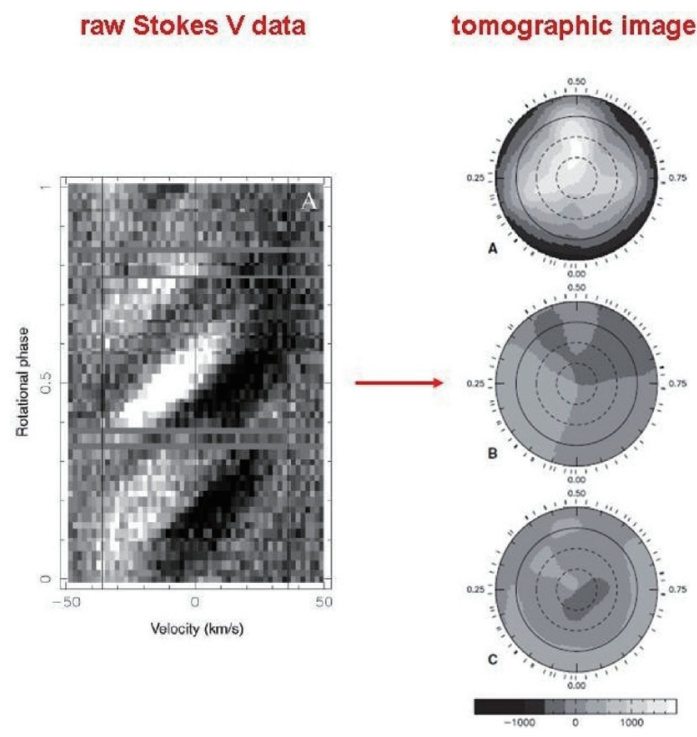

(b)

Fig. 1. (a) Early star schematic with spatially resolved linear polarization and corresponding Stokes visibilities. (b) Radial, azimuthal, and meridional magnetic field distributions of the late-type star V374 Peg. 


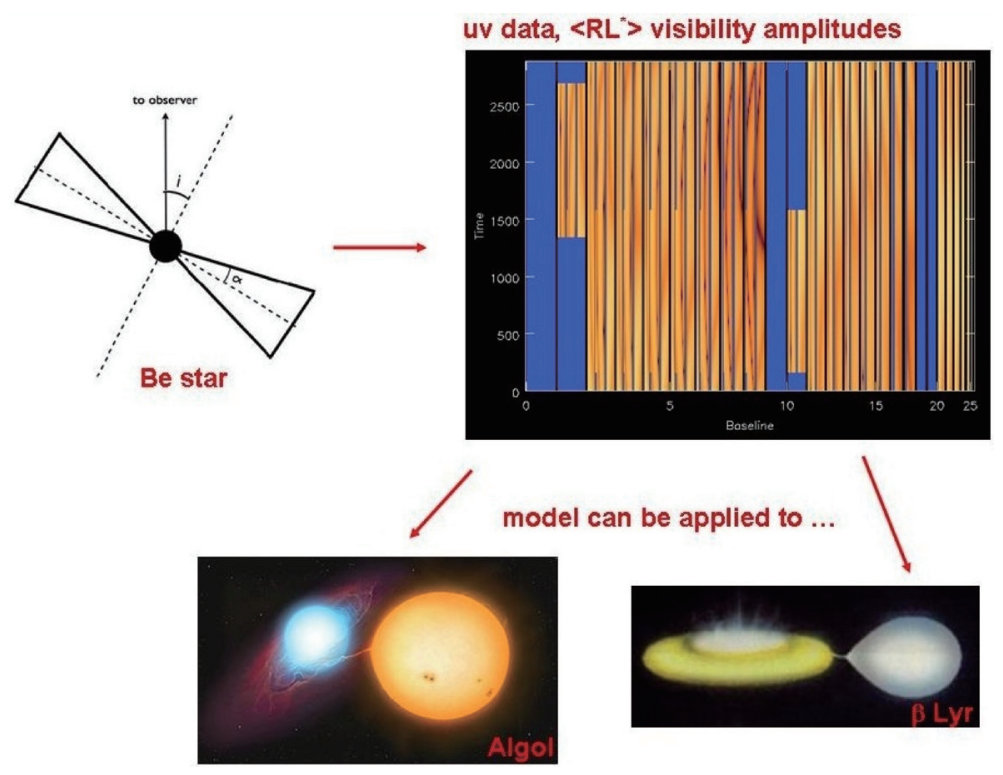

Fig. 2. The right and left circular (RL) correlations of a typical Be star at $2.2 \mu \mathrm{m}$ (Mackay et al. 2009). To first order, Be star models can be incorporated into interacting binary star models.

ing mass to an early Be-like star. Lomax et al. (2011) have completed a spectropolarimetric study of the circumbinary environment of this source. They used new and archival optical spectropolarimetry as well as filter polarimetry. When organized versus orbital phase, these data are consistent with a Thomson scattering model containing an accretion disk, stream/disk impact point, and jets offset from the Be-like component. Schmitt et al. (2009), using optical interferometry, showed that the center of light of $\beta$ Lyr's H $\alpha$ envelope moved with respect to the stars as a function of orbital phase. Zhao et al. (2008) resolved the stellar components of $\beta$ Lyr, also using optical interferometry, and derived an orbital solution of the stellar components for the first time to scale all physical parameters of the system. Clearly, future OIP observations of this object will provide a wealth of information about its circumbinary environment.

\section{BEAM COMBINER DESIGNS}

According to the Be star simulations of Mackay et al. (2009), the $1 \sigma$ errors in normalized Stokes visibilities should be less than a few times $10^{-4}$ to distinguish between competing models. This OIP science requirement is more stringent than the requirement for scalar visibilities obtained by classical optical interferometry (OIC, scalar $\sim$ Stokes I only), which in turn means that OIP beam combiner requirements must be more stringent than OIC beam combiner requirements. Note that Stokes parameter errors from classical polarimetry easily reach this level.

Amplitude and phase errors are quite significant for optical interferometry. As a matter of fact, they have much more of an effect at optical wavelengths compared to centimeter wavelengths because the variability induced by a turbulent atmosphere is much stronger at optical wavelengths. There are also slower amplitude and phase errors due to the instrument. To reach the science goals, the spectral resolution of the beam combiner should be better than 100 in order to employ coherent averaging and obtain accurate amplitudes and phases.

Spatial filtering minimizes the effects of rapid atmospheric amplitude and phase errors. The spatial filter at the input of a beam combiner can either be a pinhole or "polarization preserving" optical fibers.

This generation of optical interferometers (and possibly the next) employs mirror trains which introduce instrumental polarization that can be much larger than the polarization of an astrophysical source (Elias 2001, 2004a), perhaps up to $10 \%$. To minimize common-mode (classical, arm-based) and differential (interferometer, baseline-based) instrumental polarization, the beam combiner should use mostly refractive optics with as few reflective optics as possible or employ orthogonal pairs of reflective optics. For easier alignment, we choose the former.

Calibration will be simplified if no instrumental polar- 
ization is introduced after the polarimeter within a beam combiner. In general, instrumental polarization depends on telescope pointing. Counteracting this effect with specialized optics has been demonstrated by Rousselet-Perraut et al. (2006), but we assume instead that a polarization calibration model of the complete instrument will be created and maintained.

Like amplitude errors, random differential atmospheric phase errors occur at relatively high frequencies. They can be minimized by coherent averaging and phase bootstrapping in conjunction with closure phases (Armstrong et al. 1998, Jorgensen et al. 2007). Coherent averaging requires measurements at multiple wavelengths, and this has been demonstrated with Navy Prototype Optical Interferometer data.

Dispersing the fringes on a low-read-noise chargecoupled device is a logical choice for coherent averaging. Phase boot-strapping requires simultaneous measurements using non-polarimeter channels (same wavelengths as the science channels) on multiple baselines (some must be relatively short). Existing interferometers and their fringe trackers can accommodate this requirement (and also provide photometric normalization, if necessary). Slowly changing instrumental phase errors can be removed as part of imaging processing.

Taking these requirements into account, we created a preliminary spectrally dispersed image-plane polarization beam combiner (Fig. 3). The only reflecting optics used in the entire design feed the instrument. Different polarimeter configurations can be substituted as desired. This beam combiner is designed for sequential measurements of a single baseline. The spectral resolution can be changed with different wedges, and the design can be expanded for simultaneous measurements and/or multiple baselines.

We are also in the process of designing a beam combiner with simultaneous measurements of all polarimeter states. For each telescope input, it splits the light into orthogonal linear components and sends the light through fiber spatial filters. The beams are then combined to pro- duce all of the zero-spacing and interferometric correlations that are eventually transformed into Stokes parameters.

\section{PRELIMINARY POLARIMETER DESIGN}

For a simultaneous beam combiner, the polarimeter is built in. The light from each telescope is split into two orthogonal states immediately before interference. These optics cannot be easily replaced. For a sequential beam combiner (Fig. 3), however, the polarizing optics can be replaced as desired. In this section, I will discuss only the polarimeter for the sequential beam combiner in the previous section.

We have a choice of using a separate polarimeter for each arm or a single polarimeter placed after beam combination. The single-polarimeter design simplifies instrument design as well as calibration, so we will employ it in the preliminary design. The polarimeter should be able to measure all Stokes parameters, not only for astrophysical use cases but to provide the best instrumental polarization calibration.

Beam combining optics must be as stable as possible to improve calibration, which means minimizing mechanical and thermal disturbances. A rotating linear polarizer introduces vibrations, so we will not use it. In addition, all polarizers possess a small optical wedge which causes the beam to wander on the detector when rotated, thus complicating calibration. To vary the polarimeter state, we will use liquid crystal (LC) retarders that apply no wedges and minimal heat.

As mentioned in the previous section, atmospheric amplitude errors occur at relatively high frequencies. They are produced by scintillation and variable extinction. The instrument must be able to normalize these effects. Simultaneous measurements of all polarimeter states will accomplish this goal. If the polarimeter states are measured sequentially, however, they must be cycled rapidly or normalized externally. We employ a sequential

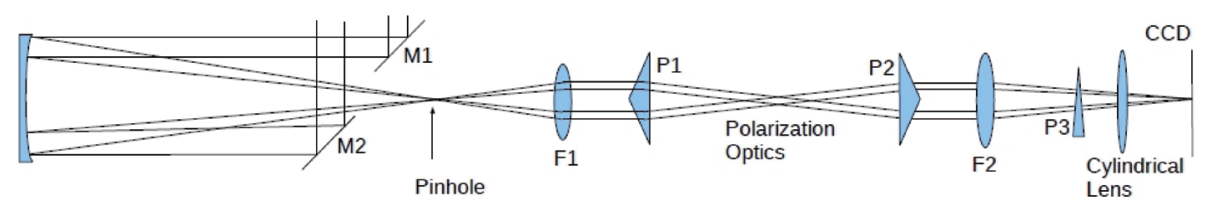

Fig. 3. Preliminary sequential polarizing beam combiner design. $M 1$ and $M 2$ are the final mirrors in the arms of the interferometer feed system. The pinhole is used as a spatial filter. F1 and F2 are conjugate lenses to collimate and focus the beams. P1 and P2 are crossing prisms. The prism P3 and cylindrical lens disperse the output. As the interferometer delay lines (not shown) are slewed across the fringes, the charge-coupled device measures their visibility versus wavelength. These measurements are performed as a function of polarimeter state. The polarization optics are described in the next section. 
instrument to simplify the optics, but we will consider a simultaneous instrument in the future.

Fig. 4 is a schematic representation of a polarimeter employing two variable LC retarders (one-wavelength range) and a fixed linear polarizer. Varying the voltage on the retarders changes the polarimeter state. Research is being performed to determine the optimum orientations, modulation patterns, and timings. Multi-output linear polarizers, such as a Wollaston, are also being considered to replace the single-output linear polarizer.

\section{END-TO-END OIP DATA REDUCTION}

We have created $2.2 \mu \mathrm{m}$ Be star models (Mackay et al. 2009). We imported them into CASA, turned them into noiseless one-second visibility samples for a scaled 27 telescope VLA-like array, and reimaged them (Elias et al. 2011). All Stokes images showed the disk, although it was

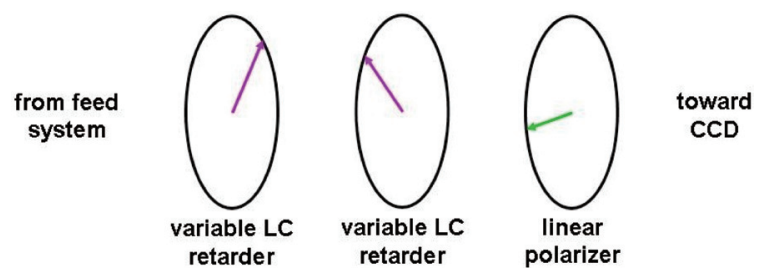

Fig. 4. Preliminary polarimeter design. None of the parts rotate. The state of the first liquid crystal (LC) retarder is varied to modulate polarization for measurement by the charge-coupled device of the optical interferometric polarimetry beam combiner (previous section). The other LC retarder does not change state. easier to see in the Stokes Q and U images because the bright and unresolved star is unpolarized.

We have recently implemented more realistic simulations, employing: 1) 9 antennas in a "Y"; 2) 15 channels from 2.1 to $2.5 \mu \mathrm{m}$; 3) Poisson noise; and 4) random phase errors, standard deviation of $10^{\circ}$. The star is B0Ve at a distance of $\sim 60 \mathrm{pc}$, which means that the Johnson $\mathrm{V}$ magnitude is $\sim 0$. The maximum baseline is longer than existing interferometers, $\sim 1,500 \mathrm{~m}$, but simulations with baselines a factor of few smaller still show the polarizing structures. Also, baselines at optical wavelengths are a factor of $\sim 3$ smaller. These two factors together set the maximum baseline well within the limits of existing interferometers and allow observations of smaller objects.

In Fig. 5b, we present a preliminary top-level design for end-to-end OIP data reduction. We will systematically write and test the software as part of a future study. The images of Fig. 5b were created as part of the imaging box in Fig. 5a.

Raw data ( $\sim$ millisecond samples), including errors, will eventually be created from the Be star models. The raw data will be written to uvfits or OI-FITS files, which are used for optical interferometry data. The filler, which has not yet been written, processes the raw data into $\sim$ onesecond uncalibrated coherence vectors and writes them into a measurement set (MS), the native CASA data format. At present, there is special software to convert the Be star model files directly to MSes with one-second averages without forming raw data.

The polarization calibration observation strategy may take a number of forms. For example, an instrument mod-

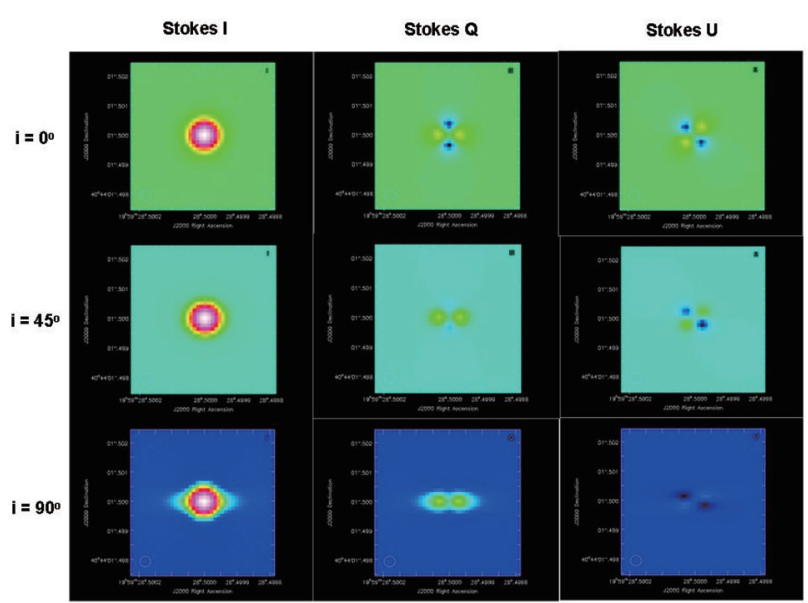

(a)

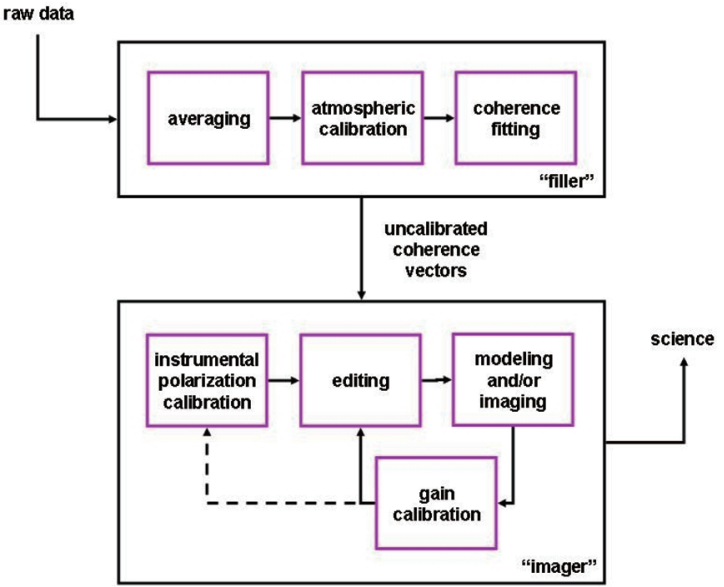

(b)

Fig. 5. (a) A Be star model reimaged using CASA. Stokes $I, Q$ and $U$ go from left to right. The top row represents an inclination of $0^{\circ}$ (face on) and the bottom row represents an inclination of $90^{\circ}$. (b) Preliminary end-to-end optical interferometric polarimetry data reduction process. 
el, including variability caused by pointing, can be fitted to observations of polarization calibrators. The model is maintained independently of science observations. Or, one may perform observations of polarization calibrators near the science targets (in both time and location on the sky). The instrumental polarization is then assumed to be the same for both source types. Regardless of strategy, the polarization calibration data must be converted to a form that the native CASA task polcal can use. This conversion software does not yet exist, and it will probably be written in the CASA environment.

CASA already possesses mature visibility viewing and editing tasks such as msview and plotms. It also has an advanced imaging task called clean. It may be configured for multifrequency synthesis, which is relevant for most optical interferometry data because they typically are obtained over wide wavelength ranges. Multiscale clean, which uses arbitrarily shaped components (as opposed to a simple delta function), is also available.

\section{CONCLUSIONS}

Steady progress has been made in the OIP field during the last decade, including algorithm development (Geisler et al. 2008), system engineering studies (Elias et al. 2004a,c), and hardware (Stee et al. 2006). Because of this progress and our hardware and software designs, we are confident that full-Stokes imaging with optical interferometers will become commonplace in the near future and provide new insights into many astrophysical objects and phenomena.

\section{ACKNOWLEDGEMENTS}

The authors gratefully acknowledge Kumar Golap, Urvashi Rao, Gregg Hallinan, and George Moellenbrock for their advice.

\section{REFERENCES}

Armstrong JT, Mozurkewich D, Rickard LJ, Hutter DJ, Benson JA, et al., The Navy Prototype Optical Interferometer, ApJ, 496, 550 (1998). http://dx.doi.org/10.1086/305365

Chandrasekhar S, Radiative transfer (Clarendon Press, Oxford, 1950).

Donati J-F, Forveille T, Collier CA, Barnes JR, Delfosse X, et al., The large-scale axisymmetric magnetic topology of a very-low-mass fully convective star, Sci, 311, 633-635 (2006). http://dx.doi.org/10.1126/science.1121102

Elias NM II, Optical interferometric polarimetry. I. Foundation, ApJ, 549, 647-668 (2001). http://dx.doi.org/10.1086/ 319046

Elias NM II, Optical interferometric polarimetry. II. Theory, ApJ, 611, 1175-1199 (2004a). http://dx.doi.org/10.1086/ 422212

Elias NM II, Significant science projects with simple sources using an optical interferometer and polarimeter, SPIE, 5432, 175-186 (2004b). http://dx.doi.org/ $10.1117 / 12.548820$

Elias NM II, Draper DW, Noecker MC, Polarization and planet-detecting nulling interferometers, SPIE, 5555, 215230 (2004c). http://dx.doi.org/10.1117/12.564219

Elias NM II, Jones CE, Schmitt HR, Jorgensen AM, Ireland MJ, et al., The case for optical interferometric polarimetry, arXiv 0811.3139 (2008).

Elias NM II, Jorgensen AM, Mozurkewich D, Jones CE, Mackay FE, et al., Imaging stars with an optical interferometer and polarimeter, in AIP Conference Proceedings 1429: Stellar Polarimetry: From Birth to Death, Madison, WI, 27-30 Jun 2011.

Geisler R, Elias NM II, Quirrenbach A, Köhler R, Tubbs RN, et al., Simulations of imperfect PRIMA fringe sensing units and calibration strategies, SPIE, 7013, 701344 (2008). http://dx.doi.org/10.1117/12.787817

Jorgensen AM, Mozurkewich D, Armstrong JT, Schmitt H, Pauls TA, et al., Improved coherent integration through fringe model fitting, AJ, 134, 1544-1550 (2007). http:// dx.doi.org/10.1086/519379

Lomax JR, Hoffman JL, Elias NM II, Bastien FA, Holenstein $\mathrm{BD}$, Geometrical constraints on the hot spot in beta Lyrae, submitted to ApJ (2011).

Mackay FE, Elias NM II, Jones CE, Sigut TAA, Using opti$\mathrm{cal} /$ near-infrared interferometric polarimetry to place constraints on the disks surrounding Be stars, ApJ, 704, 591-605 (2009). http://dx.doi.org/10.1088/0004$637 X / 704 / 1 / 591$

Mourard D, Antonelli P, Blazit A, Bonneau D, Bresson Y, et al., VEGA: a Visible spectrograph and polarimeter for the VLTI, in the power of optical/IR interferometry: recent scientific results and 2nd generation instrumentation: Proceedings of the ESO Workshop held in Garching, Germany, 4-8 April 2005. ESO Astrophysics Symposium 395, eds. Richichi A, Delplancke F, Paresce F, Chelli A (Springer, Berlin, 2008), 395.

Quirrenbach A, Bjorkman KS, Bjorkman JE, Hummel CA, Buscher DF, et al., Constraints on the geometry of circumstellar envelopes: optical interferometric and spec- 
tropolarimetric observations of seven Be stars, ApJ, 479, 477-496 (1997). http://dx.doi.org/10.1086/303854

Rousselet-Perraut K, Le Bouquin JB, Mourard D, Vakili F, Chesneau O, et al., First sky validation of an optical polarimetric interferometer, A\&A, 451, 1133-1137 (2006). http://dx.doi.org/10.1051/0004-6361:20054296

Schmitt HR, Pauls TA, Tycner C, Armstrong JT, Zavala RT, et al., Navy prototype optical interferometer imaging of line emission regions of $\beta$ Lyrae using differential phase referencing, ApJ, 691, 984-996 (2009). http://dx.doi.org/
10.1088/0004-637X/691/2/984

Stee P, Mourard D, Bonneau D, Berlioz-Arthaud P, Domiciano de Souza A, et al., VEGA: a visible spectrograph and polarimeter for CHARA--science cases description, SPIE, 6268, 62683R (2006). http://dx.doi.org/ $10.1117 / 12.684479$

Zhao M, Gies D, Monnier JD, Thureau N, Pedretti E, et al., First resolved images of the eclipsing and interacting binary $\beta$ Lyrae, ApJ, 684, 95-98 (2008). http://dx.doi.org/ 10.1086/592146 